Homology, Homotopy and Applications, vol.15(2), 2013, pp.173-184

\title{
THE CELLULARIZATION PRINCIPLE FOR QUILLEN ADJUNCTIONS
}

\author{
J. P. C. GREENLEES AND B. SHIPLEY
}

(communicated by Daniel Dugger)

\begin{abstract}
The Cellularization Principle states that under rather weak conditions, a Quillen adjunction of stable model categories induces a Quillen equivalence on cellularizations provided there is a derived equivalence on cells. We give a proof together with a range of examples.
\end{abstract}

\section{Introduction}

The purpose of this paper is to publicize a useful general principle when comparing model categories: whenever one has a Quillen adjunction

$$
F: \mathbb{M} \rightleftarrows \mathbb{N}: U
$$

comparing two stable model categories, we obtain another Quillen adjunction by cellularizing the two model categories with respect to corresponding objects. Furthermore we obtain a Quillen equivalence provided the cells are small and the derived unit or counit is an equivalence on cells. In this case, the cellularization of the adjunction induces a homotopy category level equivalence between the respective localizing subcategories. The hypotheses are mild, and the statement may appear like a tautology. The Cellularization Principle can be directly compared to another extremely powerful formality, that a natural transformation of cohomology theories that is an isomorphism on spheres is an equivalence.

This result was first proved in an appendix of the original versions of [4], but the range of cases where the conclusion is useful led us to present the result separately from that particular application.

The paper is layed out as follows: in Section 2 we give the statement and proof of the Cellularization Principle, and the following sections give a selection of examples.

\footnotetext{
The first author is grateful for support under EPSRC grant number EP/H040692/1. This material is based upon work by the second author supported by the National Science Foundation under Grant No. DMS-1104396.

Received January 23, 2013, revised May 16, 2013; published on November 25, 2013.

2000 Mathematics Subject Classification: 55U35, 55P42, 55P60.

Key words and phrases: cellularization, Quillen model category, stable model category.

Article available at http://intlpress.com/HHA/v15/n2/a11 and doi:10.4310/HHA.2013.v15.n2.a11

Copyright (C) 2013, International Press. Permission to copy for private use granted.
} 


\section{Cellularization of model categories}

Throughout the paper we need to consider models for categories of cellular objects, thought of as built from a set of basic cells using coproducts and cofibre sequences. These models are usually obtained by the process of cellularization (sometimes known as colocalization or right localization) of model categories, with the cellular objects appearing as the cofibrant objects. Because it is fundamental to our work, we recall some of the basic definitions from $[8]$.

Definition 2.1. $[8,3.1 .8]$ Let $\mathbb{M}$ be a model category and $\mathcal{K}$ be a set of objects in $\mathbb{M}$. A map $f: X \rightarrow Y$ is a $\mathcal{K}$-cellular equivalence if for every element $A$ in $\mathcal{K}$ the induced map of homotopy function complexes $[8,17.4 .2] f_{*}: \operatorname{map}(A, X) \rightarrow \operatorname{map}(A, Y)$ is a weak equivalence. A cofibrant object $W$ is $\mathcal{K}$-cellular if $f_{*}: \operatorname{map}(W, X) \rightarrow \operatorname{map}(W, Y)$ is a weak equivalence for any $\mathcal{K}$-cellular equivalence $f$.

One can cellularize a right proper model category under very mild finiteness hypotheses. To avoid confusion due to the dual use of the word "cellular" we recall that a cellular model category is a cofibrantly generated model category with smallness conditions on its generating cofibrations and acyclic cofibrations [8, 12.1.1].

Proposition 2.2. [8, 5.1.1] Let $\mathbb{M}$ be a right proper, cellular model category and let $\mathcal{K}$ be a set of objects in $\mathbb{M}$. The $\mathcal{K}$-cellularized model category $\mathcal{K}$-cell- $\mathbb{M}$ exists: it has the same underlying category as $\mathbb{M}$, its weak equivalences are the $\mathcal{K}$-cellular equivalences, the fibrations the same as in the original model structure on $\mathbb{M}$, and the cofibrations are the maps with the left lifting property with respect to the trivial fibrations. The cofibrant objects are the $\mathcal{K}$-cellular objects.

Remark 2.3. Since the $\mathcal{K}$-cellular equivalences are defined using homotopy function complexes, the $\mathcal{K}$-cellularized model category $\mathcal{K}$-cell- $\mathbb{M}$ depends only on the homotopy type of the objects in $\mathcal{K}$.

It is useful to have the following further characterization of the cofibrant objects.

Proposition 2.4. [8, 5.1.5] If $\mathcal{K}$ is a set of cofibrant objects in $\mathbb{M}$, then the class of $\mathcal{K}$-cellular objects agrees with the smallest class of cofibrant ojects in $\mathbb{M}$ that contains $\mathcal{K}$ and is closed under homotopy colimits and weak equivalences.

Throughout this paper we consider stable cellularizations of stable model categories. Say that a set $\mathcal{K}$ is stable if for any $A \in \mathcal{K}$ all of its suspensions (and desuspensions) are also in $\mathcal{K}$ up to weak equivalence. That is, since the cellularization only depends on the homotopy type of elements in $\mathcal{K}$, if $A \in \mathcal{K}$, then for all $i \in \mathbb{Z}$ there are objects $B_{i} \in \mathcal{K}$ with $B_{i} \simeq \Sigma^{i} A$. In this case, for $\mathbb{M}$ a stable model category and $\mathcal{K}$ a stable set of objects, $\mathcal{K}$-cell- $\mathbb{M}$ is again a stable model category; see $[\mathbf{1}, 4.6]$. In this case, one can use homotopy classes of maps instead of the homotopy function complexes in Definition 2.1. That is, a map $f: X \rightarrow Y$ is a $\mathcal{K}$-cellular equivalence if and only if for every element $A$ in $\mathcal{K}$ the induced map $[A, X]_{*} \rightarrow[A, Y]_{*}$ is an isomorphism; see $[\mathbf{1}, 4.4]$.

Proposition 2.5. If $\mathbb{M}$ is a right proper, stable, cellular model category and $\mathcal{K}$ is stable, then $\mathcal{K}$ detects trivial objects. That is, an object $X$ is trivial in $\operatorname{Ho}(\mathcal{K}$-cell- $\mathbb{M})$, if and only if for each element $A$ in $\mathcal{K},[A, X]_{*}=0$, and this group of graded morphisms can equally be calculated in the homotopy category of $\mathbb{M}$. 
Proof. By $[\mathbf{9}, 7.3 .1]$, the set of cofibres of the generating cofibrations detects trivial objects. In this stable situation, a set of generating cofibrations is produced as follows in $[\mathbf{1}, 4.9]$. Define a set of horns on $\mathcal{K}$ by

$$
\Lambda \mathcal{K}=\left\{X \otimes \partial \Delta[n]_{+} \rightarrow X \otimes \Delta[n]_{+} \mid n \geqslant 0, X \in \mathcal{K}\right\}
$$

where here $\otimes$ is defined using framings (see [1] , [9], or [8]). If $J$ is the set of generating acyclic cofibrations in $\mathbb{M}$, then $J \cup \Lambda \mathcal{K}$ is the set of generating cofibrations for $\mathcal{K}$-cell- $\mathbb{M}$. Since $\mathcal{K}$ is stable, the cofibres of these maps are either contractible or are weakly equivalent to objects in $\mathcal{K}$ again.

Under a finiteness condition $\mathcal{K}$ is also a set of generators. An object $K$ is small in the homotopy category (from now on simply small ${ }^{1}$ ) if, for any set of objects $\left\{Y_{\alpha}\right\}$, the natural map $\bigoplus_{\alpha}\left[K, Y_{\alpha}\right] \longrightarrow\left[K, \bigvee_{\alpha} Y_{\alpha}\right]$ is an isomorphism.

Corollary 2.6. [12, 2.2.1] If $\mathbb{M}$ is a right proper, stable, cellular model category and $\mathcal{K}$ is a stable set of small objects, then $\mathcal{K}$ is a set of generators of $\mathrm{Ho}(\mathcal{K}-$ cell- $\mathbb{M})$. That is, the only localizing subcategory containing $\mathcal{K}$ is $\operatorname{Ho}(\mathcal{K}$-cell-MI) itself.

Our main theorem states that given a Quillen pair, appropriate cellularizations of model categories preserve Quillen adjunctions and induce Quillen equivalences.

Theorem 2.7. (The Cellularization Principle.) Let $\mathbb{M}$ and $\mathbb{N}$ be right proper, stable, cellular model categories with $F: \mathbb{M} \rightarrow \mathbb{N}$ a left Quillen functor with right adjoint $U$. Let $Q$ be a cofibrant replacement functor in $\mathbb{M}$ and $R$ a fibrant replacement functor in $\mathbb{N}$.

1. Let $\mathcal{K}=\left\{A_{\alpha}\right\}$ be a set of objects in $\mathbb{M}$ with $F Q \mathcal{K}=\left\{F Q A_{\alpha}\right\}$ the corresponding set in $\mathbb{N}$. Then $F$ and $U$ induce a Quillen adjunction

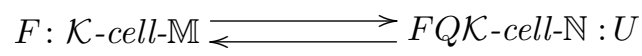

between the $\mathcal{K}$-cellularization of $\mathbb{M}$ and the $F Q \mathcal{K}$-cellularization of $\mathbb{N}$.

2. If $\mathcal{K}=\left\{A_{\alpha}\right\}$ is a stable set of small objects in $\mathbb{M}$ such that for each $A$ in $\mathcal{K}$ the object $F Q A$ is small in $\mathbb{N}$ and the derived unit $Q A \rightarrow U R F Q A$ is a weak equivalence in $\mathbb{M}$, then $F$ and $U$ induce a Quillen equivalence between the cellularizations:

$$
\mathcal{K}-\text { cell }-\mathbb{M} \simeq{ }_{Q} F Q \mathcal{K}-\text { cell }-\mathbb{N} .
$$

3. If $\mathcal{L}=\left\{B_{\beta}\right\}$ is a stable set of small objects in $\mathbb{N}$ such that for each $B$ in $\mathcal{L}$ the object $U R B$ is small in $\mathbb{M}$ and the derived counit $F Q U R B \rightarrow R B$ is a weak equivalence in $\mathbb{N}$, then $F$ and $U$ induce a Quillen equivalence between the cellularizations:

$$
U R \mathcal{L}-\text { cell }-\mathbb{M} \simeq_{Q} \mathcal{L} \text {-cell- } \mathbb{N} .
$$

Proof. Using the equivalences in $[\mathbf{8}, 3.1 .6]$, the criterion in $[\mathbf{8}, 3.3 .18(2)]$ (see also $[\mathbf{1 0}$, $2.2]$ ) for showing that $F$ and $U$ induce a Quillen adjoint pair on the cellularized model categories in (1) is equivalent to requiring that $U$ takes $F Q \mathcal{K}$-cellular equivalences between fibrant objects to $\mathcal{K}$-cellular equivalences. Any Quillen adjunction induces

${ }^{1}$ some authors use 'compact' for this notion 
a weak equivalence $\operatorname{map}(A, U R X) \simeq \operatorname{map}(F Q A, X)$ of the homotopy function complexes, see for example $[\mathbf{8}, 17.4 .15]$. So $f: X \rightarrow Y$ induces a weak equivalence $f_{*}$ : $\operatorname{map}(F Q A, X) \rightarrow \operatorname{map}(F Q A, Y)$ if and only if $U f_{*}: \operatorname{map}(A, U R X) \rightarrow \operatorname{map}(A, U R Y)$ is a weak equivalence. Thus in (1), $U$ preserves (and reflects) the cellular equivalences between fibrant objects. Hence, $U$ induces a Quillen adjunction on the cellularized model categories.

Similarly, $U f_{*}: \operatorname{map}(U R B, U R X) \rightarrow \operatorname{map}(U R B, U R Y)$ is a weak equivalence if and only if $f_{*}: \operatorname{map}(F Q U R B, X) \rightarrow \operatorname{map}(F Q U R B, Y)$ is. Given the hypothesis in (3) that $F Q U R B \rightarrow R B$ is a weak equivalence, it follows that $U f_{*}$ is a weak equivalence if and only if $f_{*}: \operatorname{map}(B, X) \rightarrow \operatorname{map}(B, Y)$ is. Thus, it follows in (3) that $U$ preserves (and reflects) the cellular equivalences between fibrant objects. Hence, $U$ induces a Quillen adjunction on the cellularized model categories. Note that the stability of $\mathbb{M}$, $\mathbb{N}, \mathcal{K}$ and $\mathcal{L}$ was not necessary for establishing the Quillen adjunction in (1) or (3).

We establish (2) in the next paragraph. One can make very similar arguments for (3) or one can deduce (3) from (2). To deduce (3) from (2), consider (2) applied to $\mathcal{K}=U R \mathcal{L}$. The hypothesis in (3) implies the hypothesis in (2) and thus produces a Quillen equivalence between $U R \mathcal{L}$-cell- $\mathbb{M}$ and $F Q U R \mathcal{L}$-cell- $\mathbb{M}$. The hypothesis in (3) also implies that $F Q U R \mathcal{L}$-cell- $\mathbb{M}$ and $\mathcal{L}$-cell- $\mathbb{M}$ are the same cellularization of $\mathbb{M}$. Thus, (3) follows.

We now return to the Quillen equivalence in $(2)$. Since $\mathbb{M}$ and $\mathcal{K}$ are stable, $\mathcal{K}$-cell- $\mathbb{M}$ is a stable model category by $[\mathbf{1}, 4.6]$. Since left Quillen functors preserve homotopy cofibre sequences, $F Q \mathcal{K}$, and hence also $F Q \mathcal{K}$-cell- $\mathbb{N}$, are stable. The Quillen adjunction in (1) induces a derived adjunction on the triangulated homotopy categories; we show that this is actually a derived equivalence. Both derived functors are exact (since the left adjoint commutes with suspension and cofibre sequences and the right adjoint commutes with loops and fibre sequences). As a left adjoint, $F$ also preserves coproducts. We next show that the right adjoint preserves coproducts as well.

Since $\mathcal{K}=\left\{A_{\alpha}\right\}$ detects $\mathcal{K}$-cellular equivalences, to show that $U$ preserves coproducts it suffices to show that for each $A_{\alpha} \in \mathcal{K}$ and any family $\left\{X_{i}\right\}$ of objects in $\mathbb{N}$ the natural map

$$
\left[A_{\alpha}, \bigvee_{i} U X_{i}\right] \rightarrow\left[A_{\alpha}, U\left(\bigvee_{i} X_{i}\right)\right]
$$

is an isomorphism. Using the adjunction and the fact that each $A_{\alpha}$ is small, the source can be rewritten as

$$
\left[A_{\alpha}, \bigvee_{i} U X_{i}\right] \cong \bigoplus_{i}\left[A_{\alpha}, U X_{i}\right] \cong \bigoplus_{i}\left[F Q A_{\alpha}, X_{i}\right]
$$

Similarly, using the adjunction, the target is isomorphic to $\left[F Q A_{\alpha}, \bigvee_{i} X_{i}\right]$. Since $F Q A_{\alpha}$ is assumed to be small, the source and target are isomorphic and this shows that $U$ commutes with coproducts.

Consider the full subcategory of objects $M$ in $\operatorname{Ho}(\mathcal{K}$-cell-M) (or respectively, $N$ in $\operatorname{Ho}(F Q \mathcal{K}$-cell- $\mathbb{N})$ ) such that the unit $Q M \rightarrow U R F Q M$ (or respectively, the counit $F Q U R N \rightarrow R N$ ) of the adjunction is an equivalence. Since both derived functors are exact and preserve coproducts, these are localizing subcategories. Since for each $A$ in $\mathcal{K}$ the unit is an equivalence and $\mathcal{K}$ is a set of generators by Corollary 2.6 , the unit is an equivalence on all of $\mathrm{Ho}(\mathcal{K}$-cell-M). It follows that the counit is also an 
equivalence for each object $N=F Q A$ in $F Q \mathcal{K}$. Since $F Q \mathcal{K}$ is a set of generators for $\mathrm{Ho}(F Q \mathcal{K}$-cell-N $)$, the counit is also always an equivalence. Statement (2) follows.

Note that if $F$ and $U$ form a Quillen equivalence on the original categories, then the conditions in Theorem 2.7 parts (2) and (3) are automatically satisfied. Thus, they also induce Quillen equivalences on the cellularizations.

Corollary 2.8. Let $\mathbb{M}$ and $\mathbb{N}$ be right proper, stable cellular model categories with $F: \mathbb{M} \rightarrow \mathbb{N}$ a Quillen equivalence with right adjoint $U$. Let $Q$ be a cofibrant replacement functor in $\mathbb{M}$ and $R$ a fibrant replacement functor in $\mathbb{N}$.

1. Let $\mathcal{K}=\left\{A_{\alpha}\right\}$ be a stable set of small objects in $\mathbb{M}$, with $F Q \mathcal{K}=\left\{F Q A_{\alpha}\right\}$ the corresponding set of objects in $\mathbb{N}$. Then $F$ and $U$ induce a Quillen equivalence between the $\mathcal{K}$-cellularization of $\mathbb{M}$ and the $F Q \mathcal{K}$-cellularization of $\mathbb{N}$ :

$$
\mathcal{K}-\text { cell }-\mathbb{M} \simeq{ }_{Q} F Q \mathcal{K}-\text { cell }-\mathbb{N} .
$$

2. Let $\mathcal{L}=\left\{B_{\beta}\right\}$ be a set of small objects in $\mathbb{N}$, with $U R \mathcal{L}=\left\{U R B_{\beta}\right\}$ the corresponding set of objects in $\mathbb{N}$. Then $F$ and $U$ induce a Quillen equivalence between the $\mathcal{L}$-cellularization of $\mathbb{N}$ and the $U R \mathcal{L}$-cellularization of $\mathbb{M}$ :

$$
U R \mathcal{L} \text {-cell- } \mathbb{M} \simeq_{Q} \mathcal{L} \text {-cell- } \mathbb{N} .
$$

In $[10,2.3]$ Hovey gives criteria for when localizations preserve Quillen equivalences. Since cellularization is dual to localization, a generalization of this corollary without stability or smallness hypotheses follows from the dual of Hovey's statement.

\section{Smashing localizations}

We suppose given a map $\theta: R \longrightarrow T$ of ring spectra (or DGAs). This gives the extension and restriction of scalars Quillen adjunction

$$
\theta_{*}: R-\bmod \longrightarrow T-\bmod : \theta^{*},
$$

where $\theta_{*} N=T \wedge_{R} N$. We apply Theorem 2.7 with $\mathbb{M}=R$-mod and $\mathbb{N}=T$-mod. The category of $T$-modules is generated by the $T$-module $T$, and we use that as the generating cell. The following uses the ideas of the Cellularization Principle.

Corollary 3.1. If $T \wedge_{R} T \stackrel{\simeq}{\longrightarrow} T$ is an equivalence of $R$-modules, then the Quillen pair

$$
\theta_{*}: T \text {-cell-R-modules } \longleftrightarrow \text { T-modules }: \theta^{*}
$$

induces

1. a Quillen equivalence if $\theta^{*} T$ is small as an $R$-module, or

2. in general, an equivalence of triangulated categories

$$
T \text {-loc-Ho(R-modules }) \simeq H o(T \text {-modules })
$$

where loc denotes the localizing subcategory.

Proof. In the first case with $\theta^{*} T$ small, this follows directly from Part 3, Theorem 2.7 with $\mathbb{M}=R$-modules and $\mathbb{N}=T$-modules, taking $T$ to be the generator of $\mathbb{N}$. In the second case, we again apply Part 3 . Here the hypothesis shows that the counit is a 
derived equivalence on cells. However the complication is that $\theta^{*} T$ will not usually be small as an $R$-module.

Nonetheless, the counit is still a derived equivalence for the $R$-module $T$. It remains to argue that the derived counit gives the stated equivalence. For this we note that the right adjoint preserves arbitrary sums: this is obvious if we are working with actual modules, but in general we may use the fact that $\theta^{*}$ is also a left adjoint (with right adjoint the coextension of scalars). It follows that we have an equivalence of the localizing subcategories generated by $T$ on the two sides.

For the first example, we take $R$ and $T$ to be conventional commutative rings or DGAs and $T=\mathcal{E}^{-1} R$ for some multiplicatively closed set $\mathcal{E}$. The condition is satisfied since $\mathcal{E}^{-1} R \otimes_{R} \mathcal{E}^{-1} R \cong \mathcal{E}^{-1} R$ and we find

$$
\mathcal{E}^{-1} R \text {-loc-Ho }(R \text {-modules }) \simeq \operatorname{Ho}\left(\mathcal{E}^{-1} R \text {-modules }\right) .
$$

Example 3.2. It is worth giving an example to show that we do not obtain a Quillen equivalence between $T$-cell- $R$-modules and $T$-modules in general. This shows that the smallness hypothesis in the Cellularization Principle is necessary.

For this we take $R=\mathbb{Z}$ and $T=\mathbb{Z}[1 / p]$. We note that any object $M$ in the localizing subcategory of $T$-cell- $R$-modules generated by $T$ has the property that $M \simeq M[1 / p]$. Accordingly $M=\mathbb{Z} / p^{\infty}$ is not in this localizing subcategory. On the other hand $M$ is not $T$-cellularly equivalent to 0 since $\operatorname{Hom}\left(\mathbb{Z}[1 / p], \mathbb{Z} / p^{\infty}\right) \neq 0$.

More generally any smashing localization of module spectra behaves in a similar way (see $[\mathbf{7}]$ for related discussion). We first suppose that $R$ is a cofibrant $\mathbb{S}$-algebra spectrum in the sense of [3], where $\mathbb{S}$ is the sphere spectrum; see also Remark 3.4 below. We next suppose given a smashing localization $L$ of the category of $R$-module spectra. By [3, VIII.2.2], there is a ring map $\theta: R \longrightarrow L R$ so that we may apply the above discussion with $T=L R$. The smashing condition states that we have an equivalence $L N \simeq L R \wedge_{R} N$ for any $R$-module $N$.

Proposition 3.3. Suppose given a cofibrant $\mathbb{S}$-algebra spectrum $R$ and a smashing localization $L$ on the category of $R$-modules. We have a diagram

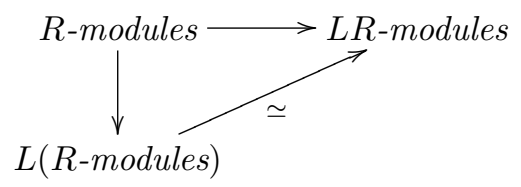

of left Quillen functors where $L(R$-modules) is the localization of the model category of $R$-modules. These induce a Quillen equivalence

$$
L(R \text {-modules }) \simeq_{Q} \text { LR-modules }
$$

and triangulated equivalences

$$
\text { LR-loc-Ho }(R \text {-modules }) \simeq H o(L(R \text {-modules })) \simeq H o(L R \text {-modules }) .
$$

Proof. The ring map $\theta: R \longrightarrow L R=T$ gives the top horizontal left Quillen functor $\theta_{*}$. The vertical map is the identity on underlying categories, which is a left Quillen functor by definition of the local model structure. The diagonal map is again $\theta_{*}$, and 
it exists because $L$ is smashing so that $L$-equivalences are taken to equivalences of $L R$-modules. This gives the diagram of left Quillen functors.

To see the diagonal is a Quillen equivalence we apply Part 3 of the Cellularization Principle. The $L R$-module $L R$ is a small generator of $L R$-modules, and the smashing condition means that $L$ ( $R$-modules) is generated by the single object $L R$, and the universal property together with the smallness of $R$ shows $L R$ is small in $L(R$-modules).

We apply Corollary 3.1 to obtain the statement about localizing categories, since the smashing condition applies to the generator $N=L R$ to show the hypotheses hold.

See also [3, VIII.3.2] and [12, 3.2(iii)].

Remark 3.4. For an arbitrary $\mathbb{S}$-algebra spectrum $T$, consider a cofibrant replacement $\theta: R \rightarrow T$ in $\mathbb{S}$-algebras and consider the relationship between smashing localizations of $R$-modules and $T$-modules. More precisely, suppose given a bifibrant $T$-module $E$ so that $L_{E}$ (localization with respect to the $E$-equivalences as in [3, VIII.1.1]) is a smashing localization on $T$-modules. In this case there is a corresponding $R$-module $F$, giving a smashing localization $L_{F}$ on $R$-modules such that the localized model categories are Quillen equivalent,

$$
L_{F}(R \text {-modules }) \simeq_{Q} L_{E}(T \text {-modules }) .
$$

In fact one can take $F$ to be the cofibrant replacement in $R$-modules of $\theta^{*} E$. Since $\theta: R \rightarrow T$ is a weak equivalence, the functors $\theta_{*}$ and $\theta^{*}$ induce a Quillen equivalence between the categories of $R$-modules and $T$-modules. Using the criteria in [10, 2.3] for when localizations preserve Quillen equivalences (dual to Corollary 2.8 above), one can show that the localization model category of $R$-modules with respect to the $F$-equivalences is Quillen equivalent to the localization model category of $T$ modules with respect to the $\theta_{*} F \cong T \wedge_{R} F$-equivalences. Since $\theta_{*} F$ and $E$ are weakly equivalent cofibrant $T$-modules, the $\theta_{*} F$-equivalences agree with the $E$-equivalences, so we have

$$
L_{F}(R \text {-modules }) \simeq_{Q} L_{\theta_{*} F}(T \text {-modules }) \cong L_{E}(T \text {-modules }) .
$$

In this situation one can show that there is a weak equivalence $T \wedge_{R} L_{F} R \simeq L_{E} T$. Using this, one can then show that if $L_{E}$ is smashing, then $L_{F}$ is also a smashing localization. Note here though that [3, VIII.2.2] only applies to $R$. So although $L_{F} R$ is constructed as an $S$-algebra, $L_{E} T$ is only constructed as a $T$-module.

Perhaps the best known example in the category of spectra is when we consider the localization of $\mathbb{S}$-modules with respect to $E_{n}$, the $n$th $p$-local Morava $E$-theory. We denote localization with respect to any spectrum weakly equivalent to $E_{n}$ by $L_{n}$. Following the remark above, we consider $R=c \mathbb{S}$, the cofibrant replacement of the sphere spectrum $\mathbb{S}$ and see that $L_{n}(\mathbb{S}$-modules $)$ and $L_{n}(c \mathbb{S}$-modules $)$ are Quillen equivalent. Proposition 3.3 then shows that

$$
L_{n}(\mathbb{S} \text {-modules }) \simeq_{Q} L_{n}(c \mathbb{S} \text {-modules }) \simeq_{Q}\left(L_{n} c \mathbb{S}\right) \text {-modules }
$$

and the homotopy categories of these are equivalent to $\left(L_{n} \mathbb{S}\right)$-loc-Ho( $\mathbb{S}$-modules $)$. 


\section{Isotropic equivalences of ring $G$-spectra}

We suppose given a map $\theta: R \longrightarrow T$ of ring $G$-spectra. This gives the extension and restriction of scalars Quillen adjunction

$$
\theta_{*}: R \text {-mod } \longleftrightarrow T \text {-mod }: \theta^{*} .
$$

We apply Theorem 2.7 Part 2 with $\mathbb{M}=R$-mod and $\mathbb{N}=T$-mod. If $G$ is the trivial group, then $R$ generates $R$-modules and the Cellularization Principle shows we have an equivalence if $\theta$ is a weak equivalence of $R$-modules.

If $G$ is non-trivial, we get a somewhat more interesting example. The category of $R$-modules is generated by the extended objects $G / H_{+} \wedge R$ as $H$ runs through closed subgroups of $G$ and the unit is the comparison $G / H_{+} \wedge \theta$. If $\mathcal{F}$ is a family of subgroups, we say $\theta$ is an $\mathcal{F}$-equivalence if $G / H_{+} \wedge \theta$ is an equivalence for all $H$ in $\mathcal{F}$. Define $\mathcal{F}$-cellularization of $R$-mod to be cellularization with respect to the set of all suspensions and desuspensions of objects $G / H_{+} \wedge R$ for $H$ in $\mathcal{F}$. Then the Cellularization Principle shows that if $\theta$ is an $\mathcal{F}$-equivalence we have a Quillen equivalence

$$
\mathcal{F} \text {-cell- } R \text {-module- } G \text {-spectra } \simeq \mathcal{F} \text {-cell- } T \text {-module- } G \text {-spectra } .
$$

\section{Torsion modules}

Let $R$ be a conventional commutative Noetherian ring and $I$ an ideal. We apply Theorem 2.7, with $\mathbb{N}$ the category of differential graded $R$-modules and $\mathbb{M}$ the category of differential graded $I$-power torsion modules. There is an adjunction

$$
i: I \text {-power-torsion- } R \text {-modules } \longleftrightarrow R \text {-modules }: \Gamma_{I}
$$

with left adjoint $i$ the inclusion and the right adjoint $\Gamma_{I}$ defined by

$$
\Gamma_{I}(M)=\left\{m \in M \mid I^{N} m=0 \text { for } N>>0\right\} .
$$

Both of these categories support injective model structures by $[\mathbf{9}, 2.3 .13]$, with cofibrations the monomorphisms and weak equivalences the quasi-isomorphisms. For torsionmodules, one needs to bear in mind that to construct products and inverse limits one forms them in the category of all $R$-modules and then applies the right adjoint $\Gamma_{I}$; see also $[\mathbf{5}, 8.6]$. With these structures the above adjunction is a Quillen adjunction.

We now consider the Cellularization Principle with $\mathbb{M}$ the $I$-power-torsion- $R$ modules and $\mathbb{N}$ the $R$-modules. If $I=\left(x_{1}, x_{2}, \ldots, x_{n}\right)$, we may form the Koszul complex $K:=K\left(x_{1}, x_{2}, \ldots, x_{n}\right)$ as the tensor product of the complexes $R \stackrel{x_{i}}{\longrightarrow} R$, noting that it is small by construction and therefore suitable for use as a cell. Since the homology of $K$ is $I$-power torsion, $K$ is equivalent to an object $K^{\prime}$ in the category

of $I$-power torsion modules, which we may take to be fibrant. We now apply the Cellularization Principle to give an equivalence

$$
\Gamma_{I} K^{\prime} \text {-cell- } I \text {-power-torsion- } R \text {-modules } \simeq K^{\prime} \text {-cell- } R \text {-modules. }
$$

It is proved in $[\mathbf{2}, 6.1]$ that the localizing subcategory generated by $R / I$ is also generated by $K \simeq K^{\prime}$. By the same proof, we see that $K^{\prime}=\Gamma_{I} K^{\prime}$ generates the category of $I$-power torsion modules and we conclude

$$
I \text {-power-torsion- } R \text {-modules } \simeq R / I \text {-cell- } R \text {-modules. }
$$




\section{Hasse equivalences}

The idea here is that if a ring (spectrum or differential graded algebra) $R$ is expressed as the pullback of a diagram of rings, the Cellularization Principle lets us build up the model category of differential graded $R$-modules from categories of modules over the terms. See also [6] for a more general treatment. We apply the standard context of Theorem 2.7 with $\mathbb{M}$ the category of $R$-modules.

\subsection{Diagrams of modules}

To describe $\mathbb{N}$ we start with a commutative diagram

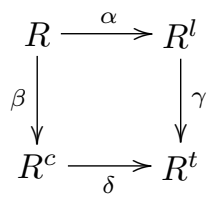

of rings.

Example 6.1. The classical Hasse principle is built on the pullback square

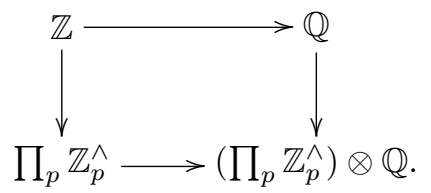

Returning to the general case, we delete $R$ and consider the diagram

$$
R^{\lrcorner}=\left(\begin{array}{r}
R^{l} \\
\left.\right|^{g} \\
v^{c} \underset{R^{t}}{\longrightarrow}
\end{array}\right)
$$

with three objects. We may form the category $\mathbb{N}=R^{\lrcorner}$-mod of diagrams

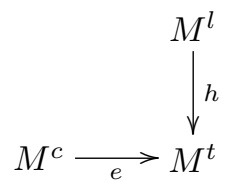

where $M^{l}$ is an $R^{l}$-module, $M^{c}$ is an $R^{c}$-module, $M^{t}$ is an $R^{t}$-module and the maps $h$ and $e$ are module maps over the corresponding maps of rings. That is, $h: M^{l} \rightarrow g^{*} M^{t}$ is a map of $R^{l}$-modules and $e: M^{c} \rightarrow d^{*} M^{t}$ is a map of $R^{c}$-modules. We will return to model structures below.

\subsection{An adjoint pair}

Since $R^{\lrcorner}$is a diagram of $R$-algebras, termwise tensor product gives a functor

$$
R^{\lrcorner} \otimes_{R}(\cdot): R \text {-mod } \longrightarrow R^{\lrcorner} \text {-mod. }
$$

Similarly, since $R$ maps to the pullback $P R^{\lrcorner}$, pullback gives a functor

$$
P: R^{\lrcorner} \text {-mod } \longrightarrow R \text {-mod. }
$$


It is easily verified that these give an adjoint pair

$$
R^{\lrcorner} \otimes_{R}(\cdot): R \text {-mod } \longleftrightarrow R^{\lrcorner}-\bmod : P .
$$

We may then consider the derived unit

$$
\eta: M \longrightarrow \underline{P}\left(R^{\lrcorner} \otimes_{R}^{L} M\right) .
$$

Since $R$ is the generator of the category of $R$-modules, we want to require that $\eta$ is an equivalence when $M=R$, which is to say the original diagram of rings is a homotopy pullback. In fact, we first fibrantly replace the original diagram of rings in the diagram-injective model category of pull-back diagrams of rings. See $[\mathbf{9}, 5.1 .3]$ or $[\mathbf{8}, 15.3 .4]$. This is discussed in more detail in $[6]$.

On the other hand, we cannot expect the counit of the adjunction to be an equivalence since we can add any module to $M^{t}$ without changing $P M^{\lrcorner}$. This is where the Cellularization Principle comes in. We should use the image of $R$ to cellularize the category of diagrams of modules. In preparation for this, we describe the model structure.

\subsection{Model structures}

We give categories of (differential graded) modules over a ring the (algebraically) projective model structure, with homology isomorphisms as weak equivalences and fibrations the surjections. The cofibrations are retracts of relative cell complexes, where the spheres are shifted copies of $R$. The category $R^{\lrcorner}$-mod gets the diagraminjective model structure in which cofibrations and weak equivalences are maps which have this property objectwise; the fibrant objects have $\gamma$ and $\delta$ surjective. This diagram-injective model structure is shown to exist for more general diagrams of ring spectra in an appendix of the original versions of [4], see also [6], and the same proof works for DGAs.

\subsection{The Quillen equivalence}

Since extension of scalars is a left Quillen functor for the (algebraically) projective model structure for any map of DGAs, $R^{\lrcorner} \otimes_{R}$ - preserves cofibrations and weak equivalences and is therefore also a left Quillen functor. We then apply the Cellularization Principle to obtain the following result.

Proposition 6.2. Assume given a commutative square of DGAs which is a homotopy pullback. The adjunction induces a Quillen equivalence

$$
R-\bmod \stackrel{\simeq}{\longrightarrow} R^{\lrcorner} \text {-cell- } R^{\lrcorner} \text {-mod, }
$$

where cellularization is with respect to the image, $R^{\lrcorner}$, of the generating $R$-module $R$.

Proof. We apply Theorem 2.7, which states that if we cellularize the model categories with respect to corresponding sets of small objects, we obtain a Quillen adjunction.

In the present case, we cellularize with respect to the single small $R$-module $R$ on the left, and the corresponding diagram $R^{\lrcorner}$on the right. First we verify that $R^{\lrcorner}$is small. Consider the three evaluation functors from $R^{\lrcorner}$-modules down to modules over the rings $R^{l}, R^{c}$, or $R^{t}$ and the associated left adjoints of these evaluation functors 
$L^{l}, L^{c}$, and $L^{t}$. The $R^{\lrcorner}$-module $R^{\lrcorner}$is the pushout of the following diagram.

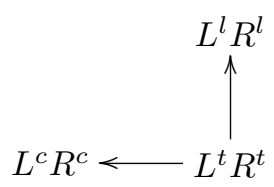

Indeed, one may explicit: $L^{l} R^{l}$ is $R^{\lrcorner}$with $R^{c}$ replaced by $0, L^{c} R^{c}$ is $R^{\lrcorner}$with $R^{l}$ replaced by 0 , and $L^{t} R^{t}$ is $R^{\lrcorner}$with $R^{l}$ and $R^{c}$ replaced by 0 . This diagram is also a homotopy pushout diagram between objects which are cofibrant in the diagraminjective model structure on $R^{\lrcorner}$-modules. It follows that $R^{\lrcorner}$is small in the homotopy category of $R^{\lrcorner}$-modules.

Since the original diagram of rings is a homotopy pullback, the unit of the adjunction is an equivalence for $R$, and we see that the generator $R$ and the generator

$R^{\lrcorner}$correspond under the equivalence, as required in the hypothesis in Part (2) of Theorem 2.7.

Since $R$ is cofibrant and generates $R$-mod, cellularization with respect to $R$ has no effect on $R$-mod and we obtain the stated equivalence with the cellularization of $R^{\lrcorner}$-mod with respect to the diagram $R^{\lrcorner}$.

\section{References}

[1] D. Barnes and C. Roitzheim, Stable left and right Bousfield localisations, preprint arXiv:1204.5384 [math.AT], 2012.

[2] W. G. Dwyer and J. P. C. Greenlees Complete modules and torsion modules, American Journal of Mathematics 124 (2002) 199-220.

[3] A. D. Elmendorf, I. Kriz, M. A. Mandell, and J. P. May, Rings, modules and algebras in stable homotopy theory, Mathematical Surveys and Monographs, Vol. 47, American Mathematical Society, Providence, RI, 1997. With an appendix by M. Cole.

[4] J. P. C. Greenlees and B. E. Shipley, An algebraic model for rational torusequivariant spectra, preprint arXiv:1101.2511v3 [math.AT], 2011.

[5] J. P. C. Greenlees and B. E. Shipley, An algebraic model for free rational Gspectra for connected compact Lie groups G, Math. Z. 269 (2011), no. 1-2, 373-400.

[6] J. P. C. Greenlees and B. E. Shipley, Homotopy theory of modules over diagrams of rings, in preparation.

[7] J. Gutiérrez, On solid and rigid monoids in monoidal categories, preprint arXiv:1303.5265 [math.CT], 2013.

[8] P. S. Hirschhorn Model categories and their localization, Mathematical Surveys and Monographs, Vol. 99, American Mathematical Society, Providence, RI, 2003.

[9] M. Hovey, Model categories, Mathematical Surveys and Monographs, Vol. 63, American Mathematical Society, Providence, RI, 1999.

[10] M. Hovey, Spectra and symmetric spectra in general model categories, J. Pure Appl. Algebra 165 (2001), no. 1, 63-127. 
[11] M. Hovey, J. H. Palmieri, and N. P. Strickland, Axiomatic stable homotopy theory, Mem. Amer. Math. Soc. 128 (1997) no. 610.

[12] S. Schwede and B. Shipley, Stable model categories are categories of modules, Topology 42 (2003), no. 1, 103-153.

J. P. C. Greenlees j.greenlees@sheffield.ac.uk

School of Mathematics and Statistics, The Hicks Building, Sheffield S3 7RH, UK

B. Shipley shipleyb@uic.edu

Department of Mathematics, Statistics and Computer Science, University of Illinois at Chicago, 508 SEO m/c 249, 851 S. Morgan Street, Chicago, IL, 60607-7045, USA 